\title{
NARRATIVE REVIEW ON THE CONSUMPTION OF SOY AND DERIVATIVES RELATED TO THE RISK OF BREAST CANCER
}

Fernando C. Ramos'1, Nayara P. Almeida1', Thaynara Mercadante'

'Universidade Federal de Goiás - Goiânia (GO), Brazil.

Objective: The objective of this work is to know the studies developed about the corelation of soy food intake and its relation with the risk of breast cancer, synthesizing the most recent recommendations. Methodology: In the preparation of this narrative review some search terms were used: Breast cancer, soy food intake and risk. These terms were introduced in Brazilian and international bibliographic databases, such as SciELO - Scientific Library Online. In addition, articles were searched in international journals such as: The American Journal of Clinical Nutrition and British Journal of Cancer. Results: In the analyzed articles, what is observed is an inverse relationship between the consumption of soy and its derivatives and the risk of breast cancer. The consumption of soy and its derivatives, in a moderate amount, decreases the risk of breast cancer and, also, the percentage of recurrence of the disease, once diagnosed. The effect of isoflavanes as an antiestrogen and the anticancer effects of multiple soy components such as DNA topoisomerase inhibition, immunoinflammatory modulation and angiogenesis blockers have been pointed out in most studies as responsible for protective action. Some studies in Asian women have come to estimate 15\% percent reduction in the risk of developing breast cancer, every $10 \mathrm{mg}$ of isoflavana ingested per day. In addition, studies also showed that isoflavones had no interaction with tamoxifen in patients taking this therapy. Conclusion: This review allowed us to analyze the correlation between soy consumption and its derivatives with breast cancer risk and mortality, suggesting that moderate soy food intake is safe and potentially beneficial for women with breast cancer. This review can highlight the subject for future works. 